\title{
Hypertrophic Osteoarthropathy on Bone Scintigraphy
}

\author{
Harleen Kaur, Mitchel Muhleman, and Helena R. Balon \\ Department of Diagnostic Radiology and Molecular Imaging, Oakland University and Beaumont Hospital, Royal Oak, Michigan
}

We present the case report of a patient with a history of lung cancer in whom the typical pattern of hypertrophic osteoarthropathy was seen on bone scintigraphy. We discuss the etiologies, pathophysiology, and management of this entity.

Key Words: hypertrophic osteoarthropathy (HOA); bone scan; molecular imaging; oncology; lung cancer

J Nucl Med Technol 2018; 46:147-148

DOI: 10.2967/jnmt.117.199315

W. e present the case report of a patient with a history of lung cancer who was being evaluated by ${ }^{99 \mathrm{~m}} \mathrm{Tc}$-methylene diphosphonate scintigraphy for bone metastasis. The scan showed the railroad-track pattern of periosteal uptake typical of hypertrophic osteoarthropathy. Bone scintigraphy is a sensitive method of detecting hypertrophic osteoarthropathy, the secondary form of which is usually associated with lung cancer.

\section{CASE REPORT}

A 63-y-old man with adenocarcinoma of the right lung presented for evaluation of osseous metastatic disease. Approximately $3 \mathrm{~h}$ after injection of $851 \mathrm{MBq}$ of ${ }^{99 \mathrm{~m}} \mathrm{Tc}-$ methylene diphosphonate, anterior and posterior wholebody bone scintigraphy (Fig. 1) was performed, with additional spot views of the pelvis, lateral ribs, and skull. The bone scans demonstrated mild, diffuse, longitudinally increased radiotracer uptake along the cortices of all long bones of the upper extremities and the right lower extremity. We postulated that the unusual asymmetric involvement of the lower extremities (diffusely decreased uptake in the left leg) might be related to the patient's history of chronic peripheral vascular disease seen on CT angiography several years earlier. The railroad-track pattern of periosteal uptake is typical of hypertrophic osteoarthropathy. Radiographs of the right ankle (Fig. 2), ordered because of pain and swelling, demonstrated a periosteal reaction along the distal diaphysis of the right tibia and fibula.

Received Jul. 14, 2017; revision accepted Oct. 17, 2017.

For correspondence or reprints contact: Harleen Kaur, Beaumont Health, 3601 W. 13 Mile Rd., Royal Oak, MI 48073.

E-mail: harleen.kaur@beaumont.org

Published online Nov. 10, 2017.

COPYRIGHT (C) 2018 by the Society of Nuclear Medicine and Molecular Imaging.

\section{DISCUSSION}

Hypertrophic osteoarthropathy is a syndrome characterized by abnormal proliferation of the skin and osseous tissue at the distal extremities. Clinical features include digital clubbing, periostosis (excessive bone formation or subperiosteal new bone formation) of tubular bones, and synovial effusions. Periostosis is usually accompanied by pain on palpation of the involved area (1).

On ${ }^{99 m} \mathrm{Tc}-m e t h y l e n e$ diphosphonate bone scintigraphy, the differential diagnosis of hypertrophic osteoarthropathy includes normal variants (the lateral cortices of the tibiae often show symmetric linear uptake); shin splints, which can appear similar to hypertrophic osteoarthropathy but are confined to the tibiae; and chronic venous insufficiency,

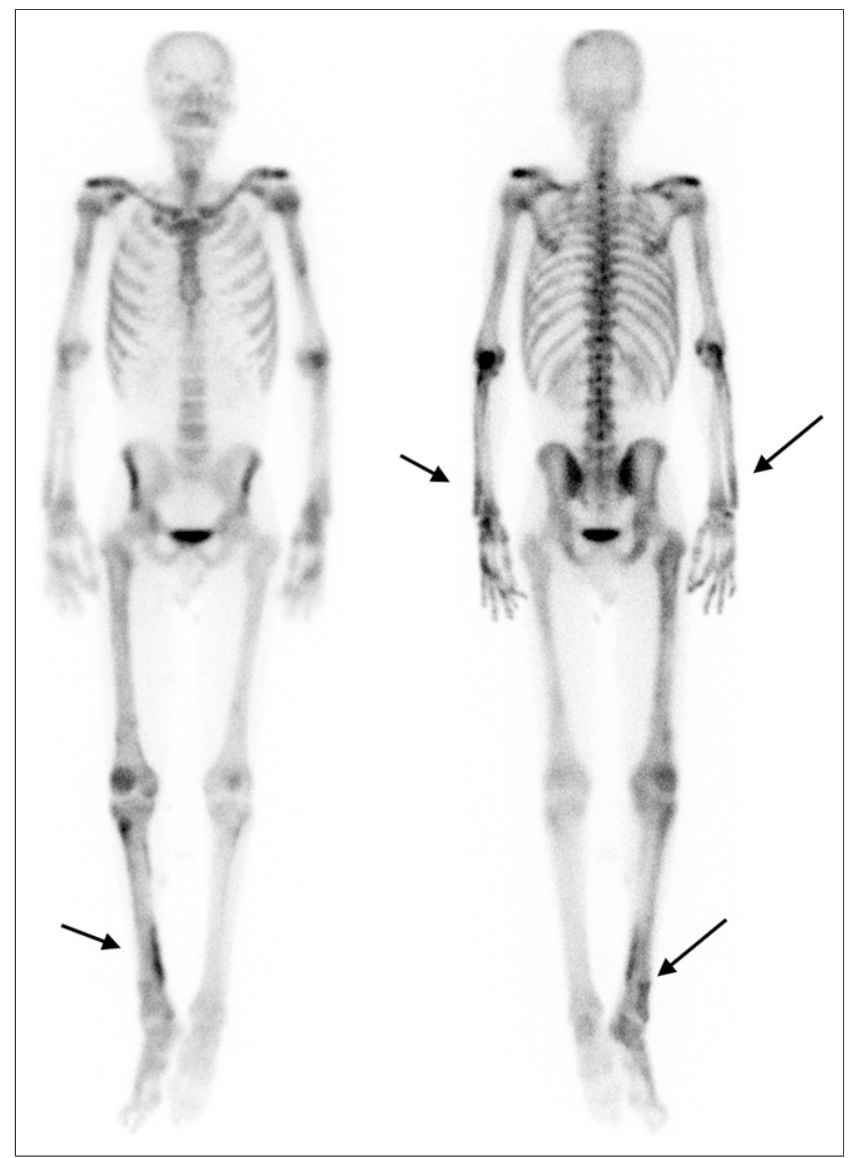

FIGURE 1. Anterior (left) and posterior (right) bone scans show increased radiotracer along cortices of all long bones in upper and lower extremities (arrows). 
FIGURE 2. Right ankle radiograph demonstrating periosteal reaction along distal tibial and fibular diaphysis (arrows).

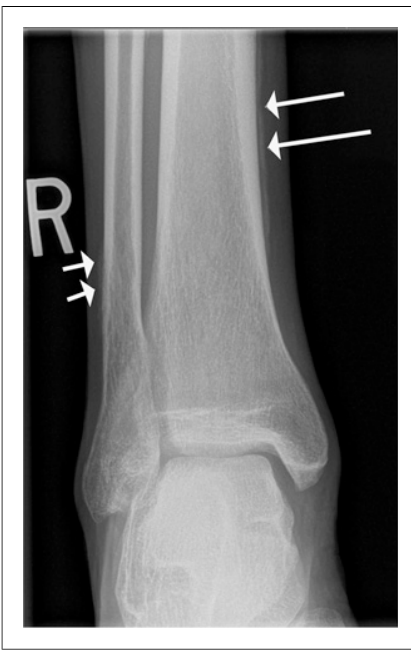

which can cause symmetric periosteal uptake, usually confined to the lower extremities below the knees. Bone scintigraphy typically demonstrates a symmetric linear increase in tracer accumulation along the diaphyseal and metaphyseal surfaces of long bones (the railroad-track pattern). In addition to being used for diagnosis, bone scintigraphy can be used to evaluate the response to therapy, as scintigraphic findings can resolve after treatment of the underlying secondary cause.

The primary form of hypertrophic osteoarthropathy is not associated with any other medical disorders. The secondary form is usually associated with lung cancer, pulmonary infections (abscess, tuberculosis, fungal infection, and pneumocystis pneumonia), chronic obstructive pulmonary disease, cystic fibrosis, and right-to-left cardiac shunts and may be seen less often in other conditions (e.g., Hodgkin lymphoma, sarcoidosis, and cirrhosis). Among patients with lung cancer, hypertrophic osteoarthropathy is associated most frequently with adenocarcinoma and least frequently with small cell carcinoma (1). Rarely, arterial vascular prosthesis infections may be associated with hypertrophic osteoarthropathy (2). Bone scintigraphy is a sensitive way to detect involvement.

The exact mechanism of hypertrophic osteoarthropathy is unclear. Currently there are 2 known pathways: neurogenic and humoral. In the neurogenic pathway, diseased organs innervated by the vagus nerve may induce a neural reflex leading to vasodilatation and increased blood flow to the extremities (3). The humoral pathway involves localized activation of the platelet-endothelial cells, with the subsequent release of fibroblast growth factors (e.g., platelet-derived growth factor) (4). The frequent association with lung disease raises the possibility that circulatory bypass of the lung may play an important role. Another hypothesis involves tumor production and release into the circulation of a factor (one possible candidate is vascular endothelial growth factor) that promotes features of hypertrophic osteoarthropathy such as vascular proliferation, edema formation, and new bone formation (4).

Treatment with nonsteroidal antiinflammatory or other analgesic medications may significantly relieve symptoms. Removal of lung cancer or treatment of the other causes of hypertrophic osteoarthropathy may cause the clinical manifestations to regress in many patients but is not always effective. In patients with refractory disease, bisphosphonates, including pamidronate (5) and zoledronic acid, have been found to be highly effective.

\section{CONCLUSION}

As illustrated by our patient who was undergoing evaluation for osseous metastatic disease from lung cancer, ${ }^{99}$ Tc-methylene diphosphonate bone scintigraphy is a sensitive method to detect hypertrophic osteoarthropathy.

\section{DISCLOSURE}

No potential conflict of interest relevant to this article was reported.

\section{REFERENCES}

1. Yap FY, Skalski MR, Patel DB, et al. Hypertrophic osteoarthropathy: clinical and imaging features. Radiographics. 2017;37:157-195.

2. Alonso-Bartolomé P, Martínez-Taboada VM, Pina T, et al. Hypertrophic osteoarthropathy secondary to vascular prosthesis infection: report of 3 cases and review of the literature. Medicine (Baltimore). 2006;85:183-191.

3. Treasure T. Hypertrophic pulmonary osteoarthropathy and the vagus nerve: an historical note. J R Soc Med. 2006;99:388-390.

4. Olán F, Portela M, Navarro C, et al. Circulating vascular endothelial growth factor concentrations in a case of pulmonary hypertrophic osteoarthropathy: correlation with disease activity. J Rheumatol. 2004;31:614-616.

5. Slobodin G, Rosner I, Feld J, et al. Pamidronate treatment in rheumatology practice: a comprehensive review. Clin Rheumatol. 2009;28:1359-1364. 